\title{
Small-angle neutron scattering study of the mixed state of $Y_{b_{3}} R_{4} S_{n}$
}

\author{
D. Mazzone, ${ }^{1}$ J. L. Gavilano, ${ }^{1}$ R. Sibille, ${ }^{2}$ M. Ramakrishnan, ${ }^{1,3}$ and M. Kenzelmann ${ }^{2}$ \\ ${ }^{1}$ Laboratory for Neutron Scattering and Imaging, Paul Scherrer Institut, 5232 Villigen PSI, Switzerland \\ ${ }^{2}$ Laboratory for Developments and Methods, Paul Scherrer Institut, 5232 Villigen PSI, Switzerland \\ ${ }^{3}$ Swiss Light Source - Condensed Matter, Paul Scherrer Institut, 5232 Villigen PSI, Switzerland \\ (Received 29 May 2014; revised manuscript received 24 June 2014; published 22 July 2014)
}

\begin{abstract}
Using the small angle neutron scattering (SANS) technique we investigated the vortex lattice (VL) in the mixed state of the stannide superconductor $\mathrm{Yb}_{3} \mathrm{Rh}_{4} \mathrm{Sn}_{13}$. We find a single domain VL of slightly distorted hexagonal geometry for field strengths between 350 and $18500 \mathrm{G}$ and temperatures between $T=0.05$ and $6.5 \mathrm{~K}$. We observe a clear in-plane rotation of the VL for different magnetic field directions relative to the crystallographic axes. We also find that the hexagonal symmetry of the VL is energetically favorable in $\mathrm{Yb}_{3} \mathrm{Rh}_{4} \mathrm{Sn}_{13}$ for external fields oriented along axes of different symmetries: twofold [110], threefold [111], and fourfold [100]. The observed behavior is different from other conventional and unconventional superconductors. The superconducting state is characterized by an isotropically gapped order parameter with an amplitude of $\Delta(0)=1.57 \pm 0.05 \mathrm{meV}$. At the lowest temperatures, the field dependence of the magnetic form factor in our material reveals a London penetration depth of $\lambda_{L}=2508 \pm 17 \AA$ and a Ginzburg coherence length of $\xi=100 \pm 1.3 \AA$, i.e., it is a strongly type-II superconductor, $\kappa=\lambda_{L} / \xi=25$.
\end{abstract}

DOI: 10.1103/PhysRevB.90.020507

PACS number(s): 74.25.Dw, 74.25.Op, 74.70.Dd

$\mathrm{Yb}_{3} \mathrm{Rh}_{4} \mathrm{Sn}_{13}$ is a member of the superconducting and/or magnetic ternary intermetallic stannides with the general formula $R_{3} M_{4} \mathrm{Sn}_{13}$, where $R$ is an alkali metal or a rare earth and $M$ is a transition metal (Ir, Rh, or Co). Several compounds of this family were first synthesized more than 30 years ago [1]. They crystallize in a cubic structure $(P m-3 n)$ with a unit cell containing 40 atoms and two formula units, $Z=2$. Because of recent observations of interesting physical phenomena for several members of this family, the 3-4-13 stannides, they recently regained interest in the research community [2,3]. For instance, $\mathrm{Ca}_{3} \mathrm{Ir}_{4} \mathrm{Sn}_{13}$ displays a superconducting phase with a $T_{c} \sim 7 \mathrm{~K}$ and a structural modulation with an onset at $T^{*} \approx 38 \mathrm{~K}$. Under pressure $p, T_{c}(p)$ reveals a dome "shaped" behavior [2]. SANS results of $\mathrm{Ca}_{3} \mathrm{Rh}_{4} \mathrm{Sn}_{13}$ showed a gradual change of the population of two structural domains with a large coexistence region at $2 \mathrm{~K}$ [3].

$\mathrm{Yb}_{3} \mathrm{Rh}_{4} \mathrm{Sn}_{13}$ is a superconductor with $T_{c}=7.4 \mathrm{~K}$ and $\mu_{0} H_{c_{2}}=2.4 \mathrm{~T}$ at $2 \mathrm{~K}$ (see Fig. 1). Compared to $\mathrm{Ca}_{3} \mathrm{Rh}_{4} \mathrm{Sn}_{13}$, the $\mathrm{Ca} 4 s$ electrons are replaced by the $\mathrm{Yb} 4 f$ electrons, introducing magnetism into play. In several superconducting materials, the $\mathrm{Yb}$ atoms are in a mixed valence state near $\mathrm{Yb}^{3+}$, as in the case of the heavy fermion systems $\alpha$ - as well as $\beta$ - $\mathrm{YbAlB}_{4}$ [4] and $\mathrm{YbNi}_{2} \mathrm{~B}_{2} \mathrm{C}$ [5]. Due to a hybridization of the $\mathrm{Yb}^{3+} f$ electrons with the conduction electrons, these materials display large Sommerfeld coefficients $\gamma$, indicating an enhancement of the effective mass of the itinerant charge carriers associated to the narrow $f$ bands $[5,6]$. Based on bond length and lattice constant analysis, it was found that the ytterbium atom in $\mathrm{Yb}_{3} \mathrm{Rh}_{4} \mathrm{Sn}_{13}$ is in a mixed valence state [7], with only a modest enhancement of $\gamma$ [8]. Magnetic susceptibility studies on the superconducting state of $\mathrm{Yb}_{3} \mathrm{Rh}_{4} \mathrm{Sn}_{13}$ revealed peaks in the magnetic response for specific fields below $H_{c_{2}}$ [8], corresponding to a broad transition into a vortex glass involving multiple steps [9].

We have carried out SANS measurements in the mixed state of $\mathrm{Yb}_{3} \mathrm{Rh}_{4} \mathrm{Sn}_{13}$ in the temperature range from $50 \mathrm{mK}$ up to $6.5 \mathrm{~K}$. The magnetic field strengths were between 350 and $18500 \mathrm{G}$ and the fields were oriented along the crystallographic [100], [110], and [111] axes. We find a single domain VL of a slightly distorted hexagonal geometry. The results are very similar for all investigated temperatures and magnetic fields above $700 \mathrm{G}$. An unexpected rotation of the VL was found for magnetic field directions along the different crystallographic axes. Our data show that the superconducting state has an isotropically gapped order parameter with an amplitude of $\Delta(0)=1.57 \pm 0.02 \mathrm{meV}$, a $T=0 \mathrm{~K}$ London penetration depth of $\lambda_{L}=2508 \pm 17 \AA$, and a Ginzburg coherence length of $\xi=100 \pm 1.3 \AA$.

Single-crystalline samples were synthesized using a $\mathrm{Sn}$ self-flux method. High-purity elements (with atomic parts of three $\mathrm{Yb}$, four $\mathrm{Rh}$, and a Sn flux) were heated up to $1050^{\circ} \mathrm{C}$ in an evacuated and sealed quartz tube. After two hours at that temperature, the liquid was cooled to $520^{\circ} \mathrm{C}$ with a cooling rate of $4{ }^{\circ} \mathrm{C} / \mathrm{h}$ and then quenched to room temperature. The excess tin flux was removed using diluted $\mathrm{HCl}$. The temperature dependence of the electrical resistivity measured on our crystals is displayed in the inset of Fig. 1. The normal-superconducting phase transition is sharp, the residual resistivity (above $T_{c}$ ) is $2.6 \mu \Omega \mathrm{cm}$ and the residual resistivity ratio $\mathrm{RRR} \approx 19$. These values show that our material has better quality than previously reported materials [9]. In contrast to $\mathrm{Ca}_{3} \mathrm{Ir}_{4} \mathrm{Sn}_{13}$, no indication for a structural modulation was found for $\mathrm{Yb}_{3} \mathrm{Rh}_{4} \mathrm{Sn}_{13}$. From our heat capacity data an electronic contribution $\gamma T$, with $\gamma=25.2 \pm 0.2 \mathrm{~mJ} / \mathrm{K}^{2} \mathrm{~mol} \mathrm{Yb}$, and a phononic contribution $\beta T^{3}$, with $\beta=1.69 \pm 0.03 \mathrm{~mJ} / \mathrm{K}^{4} \mathrm{~mol} \mathrm{Yb}$, were extracted (data not shown). The Sommerfeld coefficient $\gamma$ is roughly four times larger than for the isostructural case $\mathrm{Ca}_{3} \mathrm{Ir}_{4} \mathrm{Sn}_{13}$ [10] with the enhancement attributed to the $\mathrm{Yb} 4 f$ bands.

The SANS investigations were carried out on the instruments SANS-I and SANS-II at the neutron source SINQ at the Paul Scherrer Institute, Villigen, Switzerland. We used neutron wavelengths between 5.1 and $17.5 \AA$ with a wavelength spread of $10 \%$. The incoming neutron beam was collimated over a distance of $6 \mathrm{~m}$ (for SANS-II), $11 \mathrm{~m}$, or $18 \mathrm{~m}$ (for SANS-I). The sample was placed into a cryomagnet with a 


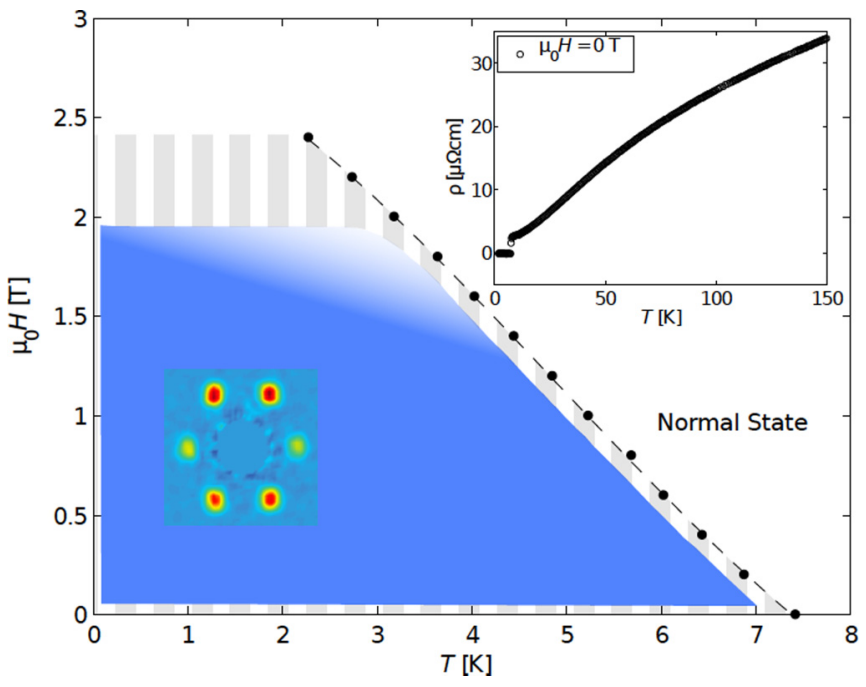

FIG. 1. (Color online) Phase diagram of the mixed state of $\mathrm{Yb}_{3} \mathrm{Rh}_{4} \mathrm{Sn}_{13}$. Electrical resistivity measurements revealed $\mu_{0} H_{c_{2}}(T)$ (black dots). The blue area indicates the region where a SANS signal was observed. In the striped area, no SANS signal was observed. (Insert) Electrical resistivity data at $\mu_{0} H=0 \mathrm{~T}$.

horizontal field parallel to the incoming neutron beam. The diffracted neutron beam was detected on a two-dimensional surface detector containing of $128 \times 128$ pixels [11]. The samples were oriented by means of $\mathrm{x}$-ray Laue diffraction and mounted on 0.5-mm-thick Al-plates of high purity. Three different samples were used in our experiments, chosen to fit the requirements of each configuration [12].

For magnetic field strengths between 700 and $18500 \mathrm{G}$, we found a single domain of a VL of nearly hexagonal structure. The obtained phase diagram is shown in Fig. 1. The hexagonal VL symmetry usually results from an isotropic crystal structure and isotropic Fermi surface (FS). The same VL geometry was found for $H$ oriented along the three main crystallographic directions ([001], [111], and [110]), for all investigated temperatures and field strengths. It is difficult to predict the symmetry of a VL for any particular material, because it depends on details of the FS. In addition, "pinning" of the flux lines often plays an important role. All this may favor any type of VL at a given field and temperature. One finds hexagonal, square or rhombic type of VL at low fields $[13,14]$. A common approach to treat the VL uses the London model corrected for nonlocal effects. The latter usually trigger phase transitions between VL structures of different symmetries. In some cases, one finds gradual changes of the structure with broad coexistence region of different VL symmetries [15]. The latter case occurs, for instance, in the isostructural compound $\mathrm{Ca}_{3} \mathrm{Rh}_{4} \mathrm{Sn}_{13}$ [3]. We observe no temperature- or field-induced phase transitions of VL, but we can not rule out nonlocal effects.

The SANS diffraction patterns for the investigated field directions at $1.8 \mathrm{~K}$ and $3500 \mathrm{G}$ are shown in Fig. 2. The extracted opening angles are $\theta_{[100]}=59.7 \pm 0.5^{\circ}, \theta_{[110]}=$ $59.9 \pm 0.7^{\circ}$ and $\theta_{[111]}=59 \pm 1^{\circ}$ where the subscripts of $\theta$ denote the crystal axis along which the external field is applied [see Fig. 2(a)]. The values of the opening angles are consistent with a nearly ideal hexagonal lattice with only a slight distortion, if any, resulting in inequivalent $k_{x}$ and $k_{y}$ directions. The elliptical axial ratio is $\eta=a / b=$ $1.07 \pm 0.03$, where $a$ and $b$ are the axes of the maximally distorted ellipsoid. Although the opening angles for fields along different crystallographic directions are roughly the same, varying the angle between the crystallographic axes and the external field results in an in-plane rotation of the VL with respect to the crystal orientation. As shown in Figs. 2(a) and 2(c), the magnetic Bragg spots for $\vec{H} \|[100]$ are turned by $22 \pm 1^{\circ}$ compared to the spots for $\vec{H} \|[110]$. In both cases, the [001] axis is vertical. To our knowledge, this rotation is rarely observed in other superconductors. A possible scenario to explain this observation may involve the explicit consideration of a rotational modulus $\mathrm{Cr}$ as in Ref. [16].

From the VL diffraction patterns, the characteristic properties of the superconducting state, such as the London penetration depth $\lambda_{L}$, the Ginzburg coherence length $\xi$ and the absolute value of the superconducting order parameter are obtained as follows. The VL of a type-II superconductor forms a two-dimensional crystal in $q$ space. The local field in the mixed state can be written as a sum over spatial Fourier components called the magnetic form factor $F\left(\vec{q}_{h, k}\right)$. The integrated intensity $I_{\text {int }}\left(\vec{q}_{h, k}\right)$ of a magnetic Bragg spot is then

$$
I_{\text {int }}\left(\vec{q}_{h, k}\right)=2 \pi V \phi_{n}\left(\frac{\mu_{n}}{4}\right)^{2} \frac{\lambda_{n}^{2}}{\Phi_{0}^{2} q_{h, k} \cos (\zeta)}\left|F\left(\vec{q}_{h, k}\right)\right|^{2},
$$

where $\mathrm{V}$ is the volume of the sample, $\phi_{n}$ is the incident neutron flux per unit area, $\lambda_{n}$ the wavelength of the incident neutron beam, $\mu_{n}=1.91$ the magnetic moment of the neutron in nuclear magnetons and $\Phi_{0}$ the flux quantum $[17,18]$. The
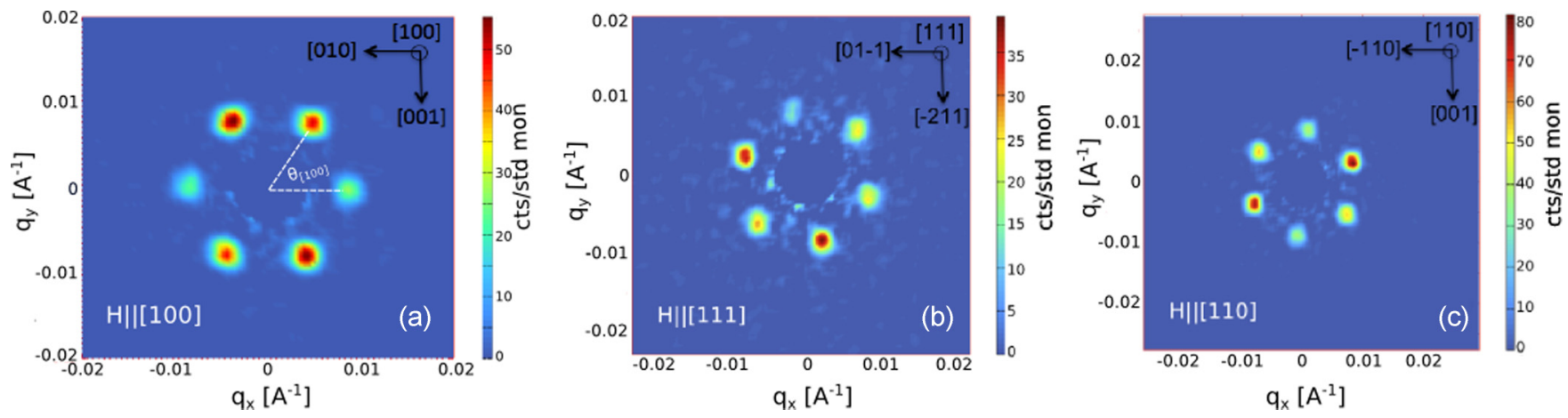

FIG. 2. (Color online) Vortex lattice diffraction pattern at $T=1.8 \mathrm{~K}$ and $\mu_{0} H=3500 \mathrm{G}$ for (a) $\vec{H} \|[100]$, (b) $\vec{H} \|[111]$, and (c) $\vec{H} \|[110]$. 

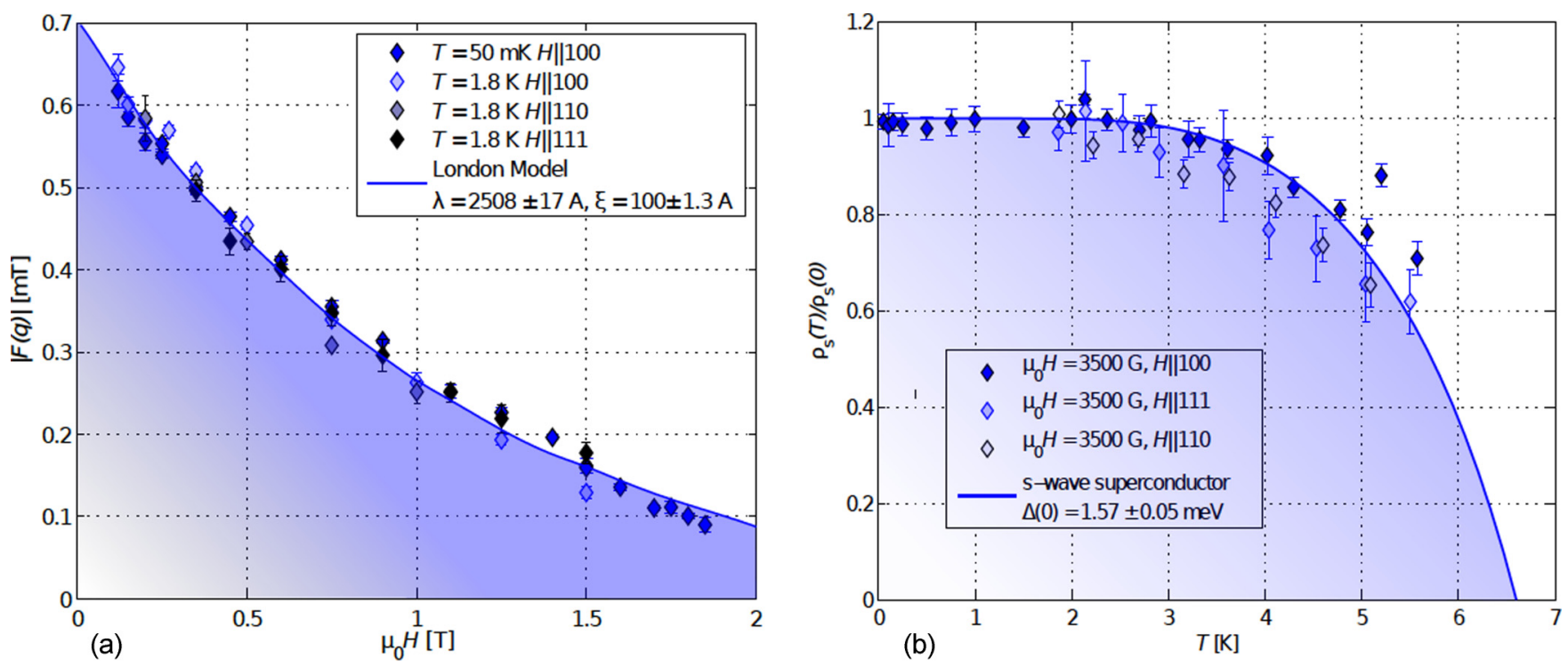

FIG. 3. (Color online) (a) Field dependence of the magnetic form factor for three crystal directions at $T=50 \mathrm{mK}$ and $1.8 \mathrm{~K}$. (b) Temperature dependence of the superfluid density. The data reveal a $s$-wave superconducting order parameter.

integrated intensity is recorded from a Gaussian fit over the rocking curve of each Bragg spot as a function of the rocking angle. This rotation is taken into account in Eq. (1) by the Lorentz factor $\cos (\zeta)$. Here, $\zeta$ represents the angle between the reciprocal lattice vector and the direction normal to both (i) the rotation axis of the cryomagnet and (ii) the incoming neutron direction. The field dependence of the magnetic form factor of $\mathrm{Yb}_{3} \mathrm{Rh}_{4} \mathrm{Sn}_{13}$ is shown in Fig. 3. There is no significant difference in the field dependence of the form factor for the different field directions relative to the crystal. This result hints for isotropic superconducting properties. Furthermore, no significant difference of the field dependent $F\left(\vec{q}_{h, k}\right)$ is observed between $50 \mathrm{mK}$ and $1.8 \mathrm{~K}$.

From the magnetic field dependence of $F\left(\vec{q}_{h, k}\right), \lambda_{L}$ and $\xi$ are obtained using a London model that takes into account the finite size of the vortex cores. The magnetic form factor is then given by [19]

$$
F\left(\vec{q}_{h, k}\right)=\frac{B e^{-0.44 q^{2} \xi^{2}}}{1+q_{h, k}^{2} \lambda_{L}^{2}}
$$

where $B$ is the applied magnetic field and $\vec{q}_{h, k}$ the fielddependent reciprocal lattice vector directly measured in our SANS experiments. The magnetic field dependence of the magnetic form factor of $\mathrm{Yb}_{3} \mathrm{Rh}_{4} \mathrm{Sn}_{13}$ was fitted using Eq. (2) with $\lambda_{L}$ and $\xi$ as free parameters. The best fit to the data reveals a London penetration depth of $\lambda_{L}=2508 \pm 17 \AA$ and a Ginzburg coherence length of $\xi=100 \pm 1.3 \AA$. From the ratio of these two values, the Ginzburg-Landau (GL) parameter $\kappa=\lambda_{L} / \xi=25 \pm 0.3$ is obtained, indicating a strong type-II superconductor. The same results are obtained for fields oriented along [100], [111], and [110] axis. Thus $\lambda_{L}$ and $\xi$ are the same along all the main directions of the crystal.

For the nonmagnetic superconductor $\mathrm{Ca}_{3} \mathrm{Rh}_{4} \mathrm{Sn}_{13}$, a GL parameter of 6.3 was inferred from SANS data $\left[\lambda_{L}(0) \approx\right.$ 841 Å] [3]. Replacing $\mathrm{Ca}$ by the rare earth $\mathrm{Yb}$ the GL parameter is increased by a factor of four. The larger London penetration depth $\left(\lambda_{L} \propto \sqrt{m^{*} / n_{s}}\right)$ of $\mathrm{Yb}_{3} \mathrm{Rh}_{13} \mathrm{Sn}_{13}$ results from an enhancement of the effective mass or a suppression of the superfluid density. This is attributed to differences of the FS of the two materials due to the $4 f \mathrm{Yb}$ bands.

We recorded several Bragg reflections at various temperatures above $T=50 \mathrm{mK}$ and found no indications for temperature-dependent changes in the position and the longitudinal FWHM $\left(\Gamma_{L}\right)$. Since the structure of the VL is at most only weakly temperature dependent, the integrated intensity of the Bragg peaks was estimated from the peak intensity and the previously determined $\Gamma_{L}$ measured at some chosen temperatures. To extract the temperature dependence of $\lambda_{L}$ from the Form factor data, we have tried two very different approximations for $\xi(T)$. (I) We assume that the coherence length follows a BCS behavior, i.e., $\xi(T)$ $=\xi(0)\left[\tanh \left(1.78 \sqrt{T_{c} / T-1}\right)\right]^{-1}$ with $\xi(0) \approx \xi(50 \mathrm{mK})$ in Eq. (2). In a second type of approximation (II) we simply neglect the temperature dependence of the coherence length. In case I, fits were implemented for $T<0.85 T_{c}$. Approximation II, of course, has stronger limitations, and here the fits were extracted to $T \leqslant 0.6 T_{c}$. In either case,

$$
\rho_{s}(T) / \rho_{s}(0)=\lambda_{L}(0)^{2} / \lambda_{L}(T)^{2},
$$

with $\lambda_{L}(0) \approx \lambda_{L}(50 \mathrm{mK})$. Data for $\rho_{s}(T) / \rho_{s}(0)$, obtained using approximation I, are shown in Fig. 3(b) for $3500 \mathrm{G}$ $(\vec{H}\|[100], \vec{H}\|[110]$, and $\vec{H} \|[111])$. Results above $T=$ $0.85 T_{c}$ are not shown and are not taken into account in the fits.

The temperature dependent superfluid density for a system with a three-dimensional Fermi surface and an isotropic $s$ wave pairing may be approximated by [20]

$$
\frac{\rho_{s}(T)}{\rho_{s}(0)}=1-\frac{1}{2 T} \int_{0}^{\infty} \cosh ^{-2}\left(\frac{\sqrt{\varepsilon^{2}+\Delta^{2}(T)}}{2 k_{B} T}\right) d \varepsilon .
$$

Here, $T$ denotes the temperature, $k_{B}$ is the Boltzman constant, $\sqrt{\varepsilon^{2}+\Delta^{2}(T)}$ the excitation energy of the superconducting state. The temperature dependence of the superconducting 


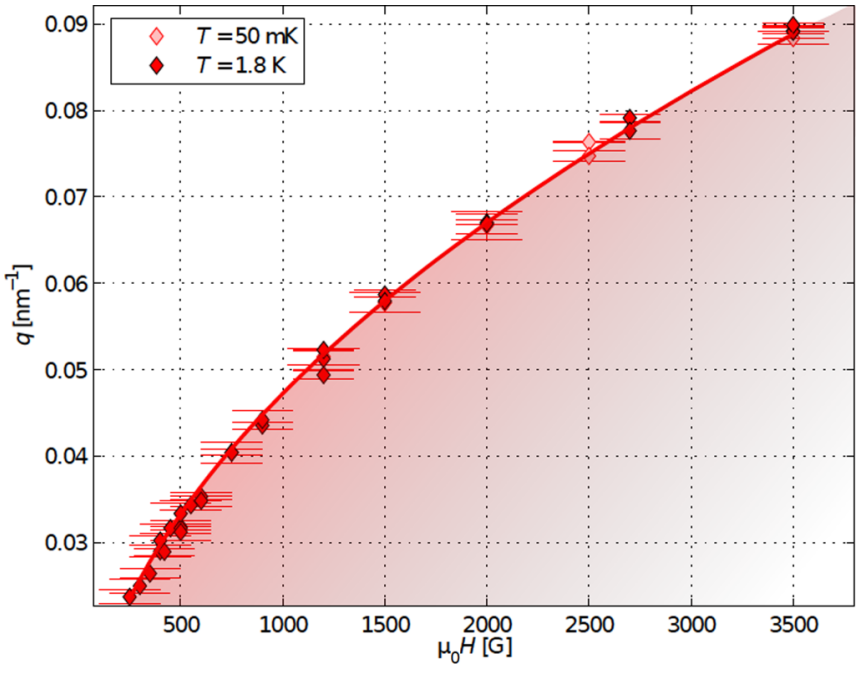

FIG. 4. (Color online) Field dependence of the $q$ vector of the VL. $q(H)$ follows a square-root behavior.

order parameter is approximated by [18]

$$
\Delta(T) \cong \Delta(0) \tanh \left(1.78 \sqrt{T_{c} / T-1}\right),
$$

where $T_{c}$ was fixed from results of thermodynamic measurements performed on the same samples (see Fig. 1). The best fit to the data using Eq. (4) and approximation I-the most reliable-yields $\Delta(0)=1.57 \pm 0.05 \mathrm{meV}$ and the coupling strength $\alpha=\Delta(0) / k_{B} T_{c}=2.8$. This value is well above the BCS weak-coupling limit of 1.76. The same results are obtained from the analysis at different fields, 3500, 7500, and $12500 \mathrm{G}$. We conclude that $\mathrm{Yb}_{3} \mathrm{Rh}_{4} \mathrm{Sn}_{13}$ is a type-II superconductor with a strong electron-phonon coupling. For completeness, we mention that using the approximation II to extract $\Delta(T)$ from Eq. (2) we find $\alpha=\Delta(0) / k_{B} T_{c}=2.1$ (data not shown). The relative difference of the extracted superconducting order parameter using approximation I and II is of the order of $25 \%$. This seems reasonable.

In our experiments, the demagnetization field $\Delta B$, estimated from the reported magnetization results [8], was less than $3 \%$ and, thus, neglected. For decreasing magnetic field strengths below approximately $7500 \mathrm{G}$, a gradual increase of the FWHM of the rocking curve $\Gamma_{L}$ is observed (see Ref. [21]). The increase of $\Gamma_{L}$ at smaller magnetic field strengths is independent of the crystal orientation. Furthermore, no evidence for a thermal-fluctuation-induced increase of $\Gamma_{L}$ was found between $50 \mathrm{mK}$ and $5 \mathrm{~K}$. Despite the increasing broadening of the magnetic Bragg reflections, their position follows the typical square-root behavior for a hexagonal VL down to magnetic field strengths of $350 \mathrm{G}$ (see Fig. 4). Therefore, we concluded that there are no temperature- or field-induced VL transitions involving changes of the geometry. The rapid increase of the width $\Gamma_{L}$ at low and high fields (data not shown) hints for a melting of the VL [21].

In summary, we have investigated the mixed state of $\mathrm{Yb}_{3} \mathrm{Rh}_{4} \mathrm{Sn}_{13}$ by means of SANS. We found a single domain of a nearly ideal hexagonal VL structure for fields between 700 and $18500 \mathrm{G}$ and temperatures between $50 \mathrm{mK}$ and $6.5 \mathrm{~K}$. Changes of the field orientation, relative to the crystal, yield no sign for a change of the symmetry of the VL. We find only an unexpected in-plane rotation of the vortex lattice for different crystal directions relative to the external field. It is unusual in a cubic system that a single VL symmetry prevails for all the field directions and field strengths. Our observations pose some challenging questions to present ideas of the VL. For instance, one expects to find some abrupt changes of the VL geometry as mandated by the hairy ball theorem [22]. The observed unusual in-plane rotation of the VL indicates that details of the "substrate" of the VL (i.e., the orientation of the crystal lattice) has to be taken explicitly into account as sketched, for instance, in Ref. [16]. Temperature fluctuations of the VL seem to play no significant role on the geometry of the $\mathrm{VL}$. $\mathrm{Yb}_{3} \mathrm{Rh}_{4} \mathrm{Sn}_{13}$ can be characterized as a type-II superconductor in the strong coupling limit with $\lambda_{L}=2508 \pm 17 \AA$ and $\xi=100 \pm 1.3 \AA$ with $\Delta(0) / k_{B} T_{c}=2.8$.

We thank the Swiss National Foundation for the support of D.M. (Project No. 200021_147071). The research leading to these results has received funding from the European Community's Seventh Framework Programme (FP7/20072013) under grant agreement no. 290605 (COFUND: PSIFELLOW). M.R. has been supported by the Erasmus Mundus program MaMaSELF. We thank J. White for helpful discussions.
[1] J. P. Remeika, G. P. Espinosa, A. S. Cooper, H. Barz, J. M. Rowell, D. B. McWhan, J. M. Vandenberg, Z. Fiske, L. D. Woolf, C. Hamaker, M. B. Maple, G. Shirane, and W. Thomlinson, Solid State Commun. 34, 923 (1980).

[2] L. E. Klintberg, S. K. Goh, P. L. Alireza, P. J. Saines, D. A. Tompsett, P. W. Logg, J. Yang, B. Chen, K. Yoshimura, and F. M. Grosche, Phys. Rev. Lett. 109, 237008 (2012).

[3] S. J. Levett, Ph.D. thesis, University of Warwick, 2003.

[4] M. Okawa, M. Matsunami, K. Ishizaka, R. Eguchi, M. Taguchi, A. Chainani, Y. Takata, M. Yabashi, K. Tamasaku, Y. Nishino, T. Ishikawa, K. Kuga, N. Horie, S. Nakatsuji, and S. Shin, Phys. Rev. Lett. 104, 247201 (2010).

[5] S. K. Dhar, R. Nagaraja, Z. Hossaina, E. Tominez, C. Godart, C. Gupta, and R. Vijayaraghavan, Solid State Commun. 98, 985 (1996).
[6] S. Nakatsuji, K. Kuga, Y. Machida, T. Tayama, T. Sakakibara, Y. Karaki, H. Ishimoto, S. Yonezawa, Y. Maeni, E. Pearson, G. G. Lonzarich, L. Balicas, H. Lee, and Z. Fisk, Nat. Phys. 4, 603 (2008).

[7] S. Miraglia, J. L. Hodeau, M. Marezio, C. Laviron, M. Ghedira, and G. P. Espinosa, J. Solid State Chem. 63, 358 (1986).

[8] H. Sato, Y. Aoki, and H. Sugawara, J. Phys. Soc. Jpn. 64, 3175 (1995).

[9] C. V. Tomy, G. Balakrishnan, and D. McK. Paul, Physica C 280, 1 (1997).

[10] K Wang and C. Petrovic, Phys. Rev. B 86, 024522 (2012).

[11] J. Kohlbrecher and W. Wagner, Appl. Cryst. 33, 804 (2000).

[12] The samples aligned with the external field and the incoming neutron beam along [001] and [111] direction were single 
crystals of 294 and $179 \mathrm{mg}$. The sample, aligned along the [110] consisted of three coaligned samples of $50 \mathrm{mg}$ each.

[13] S. Mühlbauer, C Pfleiderer, P. Böni, M. Laver, E. M. Forgan, D. Fort, U. Keiderling, and G. Behr, Phys. Rev. Lett. 102, 136408 (2009).

[14] C. D. Dewhurst and R. Cubitt, Physica B 385-386, 176 (2006).

[15] V. G. Kogan, M. Bullock, B. Harmon, P. Miranović, Lj. Dobrosavljević-Grujić, P. L. Gammel, and D. J. Bishop, Phys. Rev. B 55, R8693(R) (1997).

[16] P. Miranovic, and V. G. Kogan, Phys. Rev. Lett. 87, 137002 (2001).
[17] D. K. Christen, F. Tasset, S. Spooner, and H. A. Mook, Phys. Rev. B 15, 4506 (1977).

[18] J. S. White, R. W. Heslop, A. T. Holmes, E. M. Forgan, V. Hinkov, N. Egetenmeyer, J. L. Gavilano, M. Laver, C. D. Dewhurst, R. Cubitt, and A. Erb, Phys. Rev. B 84, 104519 (2011).

[19] M. R. Eskildsen, E. M. Forgan, and H. Kawano-Furukawa, Rep. Prog. Phys. 74, 124504 (2011).

[20] R. Prozorov and R. W. Gianetta, Supercond. Sci. Technol. 19, R41 (2006).

[21] D. Mazzone, J. L. Gavilano, R. Sibille, M. Ramakrishnan, and M. Kenzelmann, arXiv:1407.0569.

[22] M. Laver and E. M. Forgan, Nat. Commun. 1, 45 (2010). 